\title{
Factores Asociados con el Abandono de Actividades de Innovación en un País en Desarrollo: La Influencia del nivel de Cooperación Tecnológica Regional
}

\author{
Juan Fernández-Sastre ${ }^{1 *}$, Victoria Santamaría ${ }^{1}$
}

\begin{abstract}
Factors Associated with The Abandonment of Innovation Activities in A Developing Country: The Influence of the Level of Regional Technological Cooperation
\end{abstract}

Using data from the Ecuadorean Innovation Survey of 2015, this paper examines the factors associated with the abandonment of innovation activi-ties of firms in a developing country. Additionally, it is analyzed whether the influence of these factors differs depending on the level of technologi-cal cooperation existing in the regions in which the firms operate. The results indicate that the probability of abandonment is greater in firms that invest in complex innovation activities, in those that cooperate simultaneously with other firms and research institutions and in those with greater export intensity. In addition, it is observed that medium-sized firms are those that show a lower probability of abandonment. Finally, the results indicate that the influence of these factors differs considerably depending on the level of regional technological cooperation.

Keywords: abandonment of innovation activities; developing country, regional innovation systems.

Resumen: Utilizando datos de la Encuesta Ecuatoriana de Innovación del 2015, se examinan los factores asociados con el abandono de actividades de innovación de las empresas de un país en desarrollo. Adicionalmente, se analiza si la influencia de dichos factores difiere en función del nivel de cooperación tecnológica existente en las regiones en las que operan las empresas. Los resultados indican que la probabilidad de abandono es mayor en las empresas que invierten en actividades de innovación de mayor complejidad, en las que cooperan simultáneamente con otras empresas e instituciones de investigación y en las que tienen una mayor intensidad exportadora. Además, se observa que las empresas medianas son las que muestran una menor probabilidad de abandono. Finalmente, los resultados indican que la influencia de estos factores difiere considerablemente en función del nivel de cooperación tecnológica regional.

Palabras claves: abandono actividades de innovación; país en desarrollo; sistemas regionales de innovación.

Submitted: January $6^{\text {th }}, 2020 /$ Approved: May $11^{\text {th }}, 2020$

\section{Introducción}

Aunque existe una extensa literatura empírica sobre los determinantes y efectos de las actividades de innovación que desarrollan las empresas (Cohen, 1995; Griliches, 1995; Becker y Hall, 2013), la evidencia sobre los factores que influyen en el abandono de las mismas todavía es escasa (Mohne et al., 2008; Sawang y Mattews, 2010; García-Vega y López., 2010; Paunov, 2012; Cruz-Castro et al., 2017). En términos generales, estos estudios indican que las características empresariales que se asocian positivamente con el esfuerzo innovador también reducen la probabilidad de que las empresas abandonen sus proyectos de innovación. Esto es así puesto que las características de una empresa relacionadas con su capacidad para gestionar proyectos de innovación influyen tanto en sus incentivos para invertir en este tipo de proyectos como en su capacidad para llevarlos a cabo exitosamente. No obstante, toda la evidencia empírica al respecto procede de países desarrollados y, con la excepción de Paunov (2012) quien analizó los factores asociados con el abandono de actividades de innovación, durante la recesión económica del 2008, en una muestra de empresas de latinoamericanas ${ }^{1}$, no existen otros estudios para países en desarrollo, pese a que las características de la innovación y las capacidades tecnológicas difieren ampliamente entre las empresas de unos y otros países (Chaminade et al. 2009).

En los países en desarrollo, la mayoría de las empresas no tiene suficientes capacidades como para involucrarse en proyectos formales de I+D (Chaminade et al. 2009). Además, solo una pequeña porción de las empresas innovadoras recurre a relaciones de cooperación tecnológica con socios externos (Arocena y Sutz, 2001). En consecuencia, las actividades de innovación están fundamentalmente orientadas hacia la construcción de capacidades tecnológicas y la adopción e imitación de tecnologías existentes (Freeman y Soete, 1997). Por otro lado, los sistemas nacionales de innovación ${ }^{2}$ de estos países todavía están en una fase emergente (Chaminade et al., 2009), lo que reduce la capacidad que tienen las empresas para culminar con éxito sus proyectos de innovación. Por tanto, los factores que influyen en el abandono de actividades de innovación podrían diferir respecto a los que influyen en los países más avanzados.

\footnotetext{
${ }^{1}$ Nótese que este estudio no incluye empresas ecuatorianas.

${ }^{2}$ Lundvall (1992) define a los sistemas nacionales de innovación como los aspectos de la estructura económica e institucional que afectan al aprendizaje, investigación y exploración.
}

(1) Departamento de Desarrollo, Ambiente y Territorio; Facultad Latinoamericana de Ciencias Sociales, sede Ecuador - Quito, Ecuador

*Autor de correspondencia: jfernandez@flacso.edu.ec 
El presente artículo tiene como objetivo analizar los factores asociados con el abandono de actividades de innovación en un país en desarrollo como Ecuador. Ecuador es un país latinoamericano de ingreso medio-bajo que posee una estructura productiva especializada en productos de bajo valor agregado y caracterizada por un sistema de innovación todavía en una fase incipiente (Fernández y Martín, 2017). Adicionalmente, debido a que la innovación se nutre de conocimientos externos y de interacciones entre distintos agentes, que tienden a producirse dentro de un contexto geográfico (Caniels y Romijn, 2003; Cincera y Simeth, 2015), este trabajo también tiene como objetivo analizar si el nivel de cooperación tecnológica que existe en las regiones ${ }^{3}$, en las que se localizan las empresas, condiciona los factores que se asocian con el abandono de actividades de innovación. Esto es así dado que diversos estudios enfatizan el papel que tienen las relaciones de cooperación tecnológica en las actividades de innovación (Shan et al., 1994; Mowery et al., 1996). En este sentido, los factores asociados con la probabilidad de abandono, podrían resultar influenciados por el nivel de cooperación tecnológica de cada región, pues éste está relacionado con la disponibilidad y calidad del conocimiento externo al que pueden acceder las empresas para desarrollar sus proyectos tecnológicos. Aunque existe evidencia de que ciertas características regionales influyen en la probabilidad de abandono de actividades de innovación (Cruz-Castro et al., 2017), hasta la fecha no existe ningún estudio que analice cómo el nivel de cooperación tecnológica regional condiciona los factores que se asocian con el abandono de estas actividades.

El resto del artículo está organizado de la siguiente manera: la Sección 2 revisa la literatura sobre los factores que influyen en el abandono de actividades de innovación, teniendo en cuenta las particularidades de un país en desarrollo y la posible influencia del nivel de coopertación tecnológica regional. La Sección 3 describe los datos, las variables y la medología. La Sección 4 discute las implicaciones de los resultados empíricos. Finalmente, concluimos en la Sección 5.

\section{Revisión de la literatura}

\subsection{Factores asociados con el abandono de actividades de innovación}

Pese a que la inversión en actividades de innovación resulta crucial para la supervivencia de muchas empresas, numerosos proyectos de innovación son abandonados antes de su finalización (Sawang y Matthews, 2010). El abandono de estos proyectos puede llegar a afectar negativamente a los planes operacionales de las empresas (Iacovou y Dexter, 2005). Sin embargo, aunque éstas se vean forzadas a abandonar sus proyectos, su puesta en marcha puede considerarse como un proceso de aprendizaje que podría facilitar el desarrollo tecnológico en el futuro (Sawang y Matthews, 2010). No obstante e independientemente de que el abandono de actividades de innovación contribuya o no al desarrollo de capacidades para innovar, lo cierto es que unas empresas son más propensas a abandonar sus proyectos de innovación que otras (Cruz-Castro et al., 2017).
De acuerdo a la literatura, los factores que se asocian con el abandono de actividades de innovación están relacionados con aquellos que hacen que las empresas decidan invertir más recursos en actividades de innovación o ser persistentes a lo largo del tiempo en dichas inversiones (Klevorick et al., 1995; Sawang y Mattews, 2010; Tavassoli y Karlsson, 2016; Cruz-Castro et al., 2017). En este sentido, se distinguen tres grupos de factores: características de las empresas, sector económico y contexto regional (Cruz-Castro et al., 2017).

La primera característica empresarial asociada con la probabilidad de abandono es el tamaño. El tamaño de una empresa puede influir en su capacidad para desarrollar innovaciones por diversos motivos. En primer lugar, las grandes empresas cuentan con mayores recursos y acceso a financiación para sus de proyectos de innovación (Cohen, 1995) y, en ocasiones, uno de los aspectos que más influye en el abandono es la falta de recursos para poder seguir invirtiendo en actividades de innovación (Méndez-Morales, 2012). Además, las grandes empresas pueden beneficiarse de economías de escala en sus procesos de innovación, lo que influye positivamente en el desarrollo de nuevas tecnologías (Cohen, 1995). Finalmente, debido a que las grandes empresas cuentan con estructuras organizacionales que permiten una mayor división del trabajo, éstas pueden generar sinergias entre los distintos departamentos; contribuyendo así al éxito innovador (Song et al., 1997; Hage et al., 2008). No obstante, también se ha argumentado que factores como la excesiva carga burocrática de las grandes empresas y sus estructuras organizacionales para la toma de decisiones de mayor complejidad podrían propiciar el abandono de proyectos de innovación (Cohen, 1995; Cuijpers et al., 2011). En consecuencia, la relación entre el tamaño y la probabilidad de abandono puede resultar ambigua y de hecho la evidencia al respecto no es concluyente.

La segunda característica es la participación en mercados internacionales a través de la exportación. Esto se debe a que las empresas exportadoras pueden acceder a conocimientos y tecnologías no disponibles en los mercados domésticos, lo que podría facilitar el desarrollo de sus proyectos de innovación (Harris y Moffat, 2011). No obstante, si las empresas exportadoras no tienen la suficiente capacidad de absorción ${ }^{4}$, como para integrar satisfactoriamente los conocimientos provenientes del exterior, éstas podrían encontrar dificultades en el desarrollo de sus proyectos de innovación. De hecho, algunos estudios muestran que las empresas exportadoras tienen una menor probabilidad de abandono (Zouaghi y Sánchez, 2016; Cruz-Castro et al., 2017), mientras que otros revelan lo contrario (García-Vega y López, 2010; Paunov, 2012).

Otra característica individual que se asocia con la probabilidad de abandono es la edad de una empresa; dado que las empresas con más años cuentan con experiencia en el desarrollo de proyectos de innovación, lo que debería reducir la probabilidad de abandono. De hecho, por lo general, la evidencia indica que la edad de una empresa reduce la probabilidad de abandono (Paunov, 2012).

\footnotetext{
${ }^{3}$ Nótese que cuando hablamos de regiones nos referimos a las 24 provincias del Ecuador, que son unidades territoriales de segundo orden.

${ }^{4}$ Cohen y Levinthal (1990) definen la capacidad de absorción como la habilidad de una empresa para reconocer el valor de la nueva información, asimilarla y aplicarla con usos comerciales.
} 
Otro de los aspectos que podría estar asociado con la probabilidad de abandono es el tipo de actividades de innovación en las que invierten las empresas. Aquí, resulta pertinente mencionar que la innovación no solo proviene de la inversión en $\mathrm{I}+\mathrm{D}$, sino también de otro tipo de actividades como la adquisición de maquinaria, la contratación de consultorías y asistencia técnica, las actividades ingeniería y diseño industrial, las capacitaciones al personal y los estudios de mercado (Santamaría et al., 2009; Barge-Gil et al., 2011). Tal y como indican Bender y Laestadius (2004), la diferencia principal entre las actividades de I+D y las otras actividades de innovación reside en que la primera se relaciona con la generación de nuevo conocimiento y las otras con la explotación de conocimientos existentes. En este sentido, debido a las debilidades de los sistemas de innovación de los países en desarrollo y las escasas capacidades tecnológicas de sus empresas; en estos países, se observan muy pocas empresas invirtiendo en $\mathrm{I}+\mathrm{D}$ y la mayoría de los proyectos de innovación están relacionados con la inversión en otras actividades de innovación (Chaminade et al., 2009). En consecuencia, resulta pertinente analizar si el tipo de actividades de innovación en las que invierten las empresas condiciona su probabilidad de abandono. En este sentido, resulta factible considerar que las empresas, de un país en desarrollo, que invierten en actividades de $\mathrm{I}+\mathrm{D}$ muestren una mayor probabilidad de abandono que las que invierten en otro tipo de actividades de innovación; puesto que las actividades de $\mathrm{I}+\mathrm{D}$ requieren de mayores capacidades tecnológicas al igual que de un contexto científico-institucional más desarrollado (Barge-Gil et al., 2011). Por tanto, se plantea la siguiente hipótesis:

H.1. En un país en desarrollo, las empresas que invierten en $I+D$ tienen una mayor probabilidad de abandonar sus proyectos que las que invierten en otras actividades de innovación.

Otra de las características individuales que puede afectar al abandono de actividades de innovación es si dichas actividades se desarrollan conjuntamente con otras empresas o instituciones de investigación ${ }^{5}$ (Cruz-Castro et al., 2017). Esto es así dado que la cooperación con socios externos provee de acceso a conocimientos y recursos no disponibles en la empresa (Fagerberg et al., 2005). Por ende, es de esperar que las empresas que desarrollan sus proyectos con socios externos tengan una menor probabilidad de abandonarlos (Cruz-Castro et al., 2017). No obstante, para que las empresas puedan internalizar exitosamente los conocimientos externos requieren de capacidad de absorción (Cohen y Levinthal, 1990). De tal manera, que si las empresas no disponenen de la suficiente capacidad de absorcion podrían verse obligadas a abandonar sus proyectos. Además, tal y como indican Nieto y Santamaría (2007), en las relaciones de cooperación pueden emerger distintos problemas relacionados con la especificidad de los activos, con asimetrías de información entre los socios, con el posible comportamiento oportunista de alguno ellos, que pueden hacer que los proyectos de innovación sean abandonados antes de generen resultados. En este sentido, Kale et al. (2002) encontraron que el $40 \%$ de las relaciones de cooperación no culminan con el desarrollo de una nueva tecnología; mientras que Reuer y Zollo (2005) mostraron que el 34\% de las relaciones tecnológicas no culminaron exitosamente y que un $51 \%$ experimentó un resultado distinto al planificado o el retiro unilateral por parte de algún socio. Por otro lado, el éxito de un proyecto colaborativo de innovación pueden depender del tipo de socio con el que se coopera (empresas o instituciones de investigación) (Cruz-Castro et al., 2017). En este sentido, se considera que las empresas requieren de mayor capacidad de absorción para internalizar los conocimientos de las instituciones de investigación que los provenientes de otras empresas (Cohen y Levinthal, 1990). En consecuencia, es de esperar que, en los países en desarrollo, aquellas empresas que cooperan con instituciones de investigación tengan mayor probabilidad de abandono que aquellas que no cooperan o que lo hacen exclusivamente con otras empresas. Por tanto, se plantea la siguiente hipótesis:

H.2. Las empresas que cooperan con instituciones de investigación muestran una mayor probabilidad de abandono que las que no cooperan o lo hacen exclusivamente con otras empresas.

Tal y como se ha indicado, además de las características individuales, el sector económico también se asocia con la probabilidad de abandono. Esto es así puesto que las condiciones bajo las cuales se generan las innovaciones varían de sector a sector, dado que éstos difieren en términos de sus oportunidades tecnológicas ${ }^{6}$, del comportamiento de la demanda y de las condiciones de apropiación (Cohen, 1995; Becker y Hall, 2013). En este sentido es de esperar que en aquellos sectores caracterizados por mayores oportunidades tecnológicas, mejor comportamiento de la demanda y mejores condiciones de apropiación las empresas tengan una menor probabilidad de abandonar sus actividades de innovación. De igual manera, el tipo de innovación y su grado de novedad difiere entre sectores, lo que también podría influir en la probabilidad de abandono. Al respecto, Galia y Legros (2004) señalan que en los sectores de alta intensidad tecnológica, las empresas van a enfrentar mayores obstáculos y, por lo tanto, podrían tener una mayor probabilidad de abandonar sus proyectos de innovación que las que pertenecen a otros sectores.

Finalmente, otros estudios enfatizan que la probabilidad de abandono puede verse influenciada por el contexto regional; es decir, por las características del sistema regional de innovación en el que operan las empresas (Cruz-Castro et al., 2017). Esto es así porque las oportunidades tecnológicas son generadas a través de intercambios de información entre distintos agentes, que tienden a producirse dentro de un mismo territorio, dada la dificultad con la que se transmiten conocimientos tácitos a través de largas distancias (Asheim y Gertler, 2005). En este sentido, las características del sistema regional de innovación pueden influir en la probabilidad de abandono, pues la proximidad geográfica, entre los distintos agentes involucrados en el proceso innovador, genera externalidades positivas y facilita vínculos con el mercado y posibilidades de colaboración, que pueden hacer que las empresas sean más exitosas en el desarrollo de sus proyectos de

\footnotetext{
${ }^{5}$ Por instituciones de investigación nos referimos a universidades, laboratorios, empresas de I+D y organismos de ciencia y tecnología.

${ }^{6}$ Las oportunidades tecnológicas hacen referencia a la probabilidad de convertir una inversión en I+D en nuevos productos o procesos (Cohen y Levinthal, 1989; Lunn y Martin, 1986)
} 
innovación en función de la región en la que se localizan. En este sentido, Castro et al. (2017) evidenciaron que ciertas características regionales como el tamaño económico de las regiones o cómo éstas se orientan hacia la explotación de conocimientos se asociaban negativamente con la probabilidad de que las empresas españolas abandonaran sus proyectos de $\mathrm{I}+\mathrm{D}$ en un periodo de recesión económica.

\subsection{Nivel de cooperación tecnológica regional y abandono de activ- idades de innovación}

Dado que los procesos de aprendizaje, subyacentes a la innovación, se encuentran integrados en un ámbito regional (CruzCastro et al., 2017), las actividades de innovación de las empresas están largamente restringidas por las características del sistema regional de innovación en el que operan (Saxenian, 1994; Braczyk et al., 1998). En consecuencia, dado que las regiones de un país difieren en las características de sus sistemas de innovación, resulta plausible considerar que las empresas sean más o menos propensas a abandonar sus actividades de innovación en función de la región en la que desarrollan dichas actividades (Cruz-Castro et al., 2017).

Aunque la literatura empírica sugiere que varias características regionales, como la calidad del capital humano, la disponibilidad de fuentes externas de información o diversos aspectos relacionados con el ambiente político, legal y administrativo influyen en las actividades de innovación (Hemmert, 2004; Wu, 2015; Tavassoli y Karlson, 2015; López-Bazo y Motellón, 2018; Cruz-Castro et al., 2017); en este artículo, estamos interesados en analizar cómo el nivel de cooperación tecnológica regional condiciona los factores que se asocian con la probabilidad de abandono de actividades de innovación. Nuestro interés estriba en el hecho de que las actividades de innovación de las empresas dependen en gran medida de la capacidad de sus regiones para organizar procesos de aprendizaje colectivo (Gilly et al., 2014) y, por tanto, el nivel de cooperación tecnológica de cada región podría condicionar los factores que se asocian con la probabilidad de abandono. En este sentido, aunque cabría esperar que en aquellas regiones donde existe un mayor nivel de cooperación tecnológica, las empresas fuesen capaces de sobreponer los aspectos que inciden en el fracaso de sus proyectos de innovación; debido a que no existe evidencia al respecto, planteamos la siguiente pregunta de investigación:

P.1. ¿El nivel de cooperación tecnológica regional condiciona los factores que se asocian con el abandono de actividades de innovación?

\section{Datos, variables y metodología}

Este artículo utiliza datos de la Encuesta Ecuatoriana de Innovación del año $2015^{7}$ (Encuesta Nacional de Actividades de Innovación, ENAI) que abarca el periodo 2012-2014 y es llevada a cabo por el Instituto Nacional de Estadística y Censos y la Secretaría de Educación Superior, Ciencia, Tecnología e Innovación. La ENAI es una encuesta obligatoria, que sigue las directrices del Manual de Oslo, y contiene una muestra representativa sobre las actividades de innovación de 6,275 empresas que operan en los sectores de manufactura, servicios, comercio, actividades extractivas y suministros y que están localizadas en las 24 provincias del Ecuador. No obstante, dado que el objetivo de investigación es el de identificar los factores asociados con el abandono de actividades de innovación, nuestro análisis considera únicamente a las empresas innovadoras ${ }^{8}$, cuyo número asciende a 2,586 .

Dado que el objetivo es el de determinar los factores asociados con la probabilidad de actividades de innovación, nuestra variable dependiente (Abandono) es una variable dicotómica que toma valor 1 para aquellas empresas que, durante el periodo 2012-2014, tuvieron alguna actividad de innovación, para el desarrollo y/o introducción de innovaciones de producto o proceso, que fue abandonada o suspendida antes de su término y 0 para las empresas innovadoras que no abandonaron sus proyectos. Nótese que, a diferencia de otras encuestas de innovación donde el abandono de actividades de innovación hace referencia exclusivamente a las actividades de $\mathrm{I}+\mathrm{D}$, en la ENAI por actividad de innovación no solo se hace referencia a la $\mathrm{I}+\mathrm{D}$ sino que también se incluyen las siguientes actividades: adquisición de maquinaria, equipos, software y licencias, trabajos de ingeniería y desarrollo, diseño, entrenamiento y marketing.

Adicionalmente, puesto que los factores asociados con el abandono de actividades de innovación son aspectos relacionados con las características de las empresas, con el sector y con el contexto regional, las variables independientes operacionalizan dichos aspectos. En este sentido, la Tabla 1 describe cada una de las variables independientes. Por su parte el Apéndice 1 muestra una tabla de correlaciones entre las variables a nivel empresa y una serie de estadísticos descriptivos.

\footnotetext{
${ }^{7} \mathrm{http}$ //www.ecuadorencifras.gob.ec/encuesta-nacional-de-actividades-de-ciencia-tecnologia-e-innovacion-acti/

${ }^{8}$ La ENAI considera que una empresa es innovadora si ha cumplido, al menos, uno de los siguientes criterios: i) ha introducido nuevos o significativamente mejorados bienes, servicios o procesos en el mercado; ii) desarrolló o mantiene activas actividades innovadoras (incluyendo I+D); iii) abandonó actividades innovadoras.
} 
Tabla 1. Descripción de las variables independientes

\begin{tabular}{|c|c|}
\hline Nombre & Descripción \\
\hline \multicolumn{2}{|c|}{ Características de las empresas } \\
\hline Pequeña & $\begin{array}{l}\text { Variable dicotómica que toma valor } 1 \text { si la empresa tuvo en } 2012 \text { menos de } 50 \text { empleados y } 0 \text { en caso contrario. Nótese que la } \\
\text { variable Pequeña es la categoría de referencia en nuestras estimaciones. }\end{array}$ \\
\hline Mediana & Variable dicotómica que toma valor 1 si la empresa tuvo en 2012 entre 50 y 450 empleados y 0 en caso contrario. \\
\hline Grande & Variable dicotómica que toma valor 1 si la empresa tuvo en 2012 más de 450 empleados y 0 en caso contrario. \\
\hline Solo_id & $\begin{array}{l}\text { Variable dicotómica que toma valor } 1 \text { si la empresa invirtió en el } 2012 \text { en I+D interna, pero no invirtió en otras actividades de } \\
\text { innovación y } 0 \text { en caso contrario. }{ }^{*}\end{array}$ \\
\hline Solo_otros & $\begin{array}{l}\text { Variable dicotómica que toma valor } 1 \text { si la empresa invirtió en el } 2012 \text { en otras actividades de innovación, pero no invirtió en } \\
\text { I+D interna y } 0 \text { en caso contrario. * }\end{array}$ \\
\hline Id_otros & $\begin{array}{l}\text { Variable dicotómica que toma valor } 1 \text { si la empresa invirtió en } 2012 \text { en I+D interna y en otras actividades de innovación y } 0 \text { en } \\
\text { caso contrario. }{ }^{*}\end{array}$ \\
\hline Coop_instituciones & $\begin{array}{l}\text { Variable dicotómica que toma valor } 1 \text { si, durante el periodo 2012-2014, la empresa cooperó en alguna actividad de innovación } \\
\text { (I+D, ingeniería y diseño, capacitación, asistencia técnica, información, pruebas de productos y financiamiento) con institucio- } \\
\text { nes de investigación (consultores, universidades, laboratorios y empresas de I+D, organismos públicos de ciencia y tecnología } \\
\text { pero no cooperó con otras empresas y } 0 \text { en caso contrario. }\end{array}$ \\
\hline Coop_ambos & $\begin{array}{l}\text { Variable dicotómica que toma valor } 1 \mathrm{si} \text {, durante el periodo } 2012-2014 \text {, la empresa cooperó simultáneamente con empresas e } \\
\text { instituciones de investigación en alguna actividad de innovación y } 0 \text { en caso contrario. }\end{array}$ \\
\hline \multicolumn{2}{|l|}{ Sectores } \\
\hline Baja_industria & $\begin{array}{l}\text { Variable dicotómica que toma valor } 1 \text { si la empresa pertenece a un sector manufacturero de baja intensidad tecnológica y } 0 \text { en } \\
\text { caso contrario. De acuerdo a la clasificación sectorial ciiu 2-dígitos se incluyen los siguientes sectores: C10-C18, C31 y C32. }\end{array}$ \\
\hline Media_baja_industria & $\begin{array}{l}\text { Variable dicotómica que toma valor } 1 \text { si la empresa pertenece a un sector manufacturero de media-baja intensidad tecnológica } \\
\text { y } 0 \text { en caso contrario. De acuerdo a la clasificación sectorial ciiu 2-dígitos se incluyen los siguientes sectores: C19, C23-C25, } \\
\text { C33 y S95. }\end{array}$ \\
\hline Servicios_conocimiento & $\begin{array}{l}\text { Variable dicotómica que toma valor } 1 \text { si la empresa pertenece a un sector de servicios intensivo en conocimiento y } 0 \text { en caso } \\
\text { contrario. De acuerdo a la clasificación sectorial ciiu 2-dígitos se incluyen los siguientes sectores: J58, J59, J60-J63, K64-K66, } \\
\text { M69-M72, M74, M75, N80, P85, Q86-Q88, R90-R93. }\end{array}$ \\
\hline Proveedores & $\begin{array}{l}\text { Variable dicotómica que toma valor } 1 \text { si la empresa pertenece a un sector proveedor de servicios y } 0 \text { en caso contrario. De acuer- } \\
\text { do a la clasificación sectorial ciiu 2-dígitos se incluyen los siguientes sectores: D35, E36-E39 }\end{array}$ \\
\hline Extractivas & $\begin{array}{l}\text { Variable dicotómica que toma valor } 1 \text { si la empresa pertenece a un sector extractivo y } 0 \text { en caso contrario. De acuerdo a la clasi- } \\
\text { ficación sectorial ciiu 2-dígitos se incluyen los siguientes sectores: B05-B08 }\end{array}$ \\
\hline Construcción & $\begin{array}{l}\text { Variable dicotómica que toma valor } 1 \text { si la empresa pertenece al sector de la construcción y } 0 \text { en caso contrario. De acuerdo a } \\
\text { la clasificación sectorial ciiu 2-dígitos se incluyen los siguientes sectores: F41-F43 }\end{array}$ \\
\hline \multicolumn{2}{|l|}{ Regiones } \\
\hline "Región" & $\begin{array}{l}\text { Se incluyen } 24 \text { variables dicotómicas que toman valor } 1 \text { si la empresa se encuentra localizada en una de las } 24 \text { provincias del } \\
\text { Ecuador y } 0 \text { en caso contrario }\end{array}$ \\
\hline
\end{tabular}

(Nota: ${ }^{\star}$ Otras actividades de innovación, que no son I+D, incluyen las siguientes actividades: adquisición de maquinaria y equipo, adquisición de hardware, adquisición de software, adquisición de tecnología desincorporada, contratación de consultorías y asistencia técnica, actividades de ingeniería y diseño industrial, capacitación del personal y estudios de mercado.) 
Nótese que las variables Solo_id, Solo_otros e Id_otros, que muestran en qué tipo de actividades de innovación invirtieron las empresas (solo en I+D, solo en otras actividades de innovación o en ambos tipos), permiten verificar la hipótesis H.1, que consideraba que las empresas que invierten en $\mathrm{I}+\mathrm{D}$ tienen mayor probabilidad de abandono que las que invierten en otras actividades de innovación. Téngase en cuenta que la categoría de referencia de estas variables, sobre la que se interpretarán los coeficiente, son las empresas que introdujeron innovaciones sin invertir en actividades de innovación. Adicionalmente, las variables Coop_empresas, Coop_instituciones y Coop_ambos, que muestran el tipo de socios con los que cooperaron las empresas (solo con empresas, solo con instituciones o con ambos tipos de socios; respectivamente), permiten verificar la hipótesis H.2, que planteaba que las empresas que cooperan con instituciones de investigación muestran una mayor probabilidad de abandono que no cooperan o que cooperan con otras empresas. Nótese que la categoría de referencia de estas variables son las empresas innovadoras que no cooperaron.

Respecto a la metodología de estimación, denótese el abandono de una actividad de innovación de una empresa por, entonces el abandono de una actividad de innovación puede ser capturado por un modelo de elección binaria:

$$
y_{i}=\left\{\begin{array}{c}
1 \text { if } y_{i t}^{*} \geq 0 \\
0 \text { todo lo demás }
\end{array}\right.
$$

Donde la variable latente representa la propensión de una empresa a abandonar una actividad de innovación en el periodo 2012-2014, que es una función de las características individuales de las empresas, de la categoría sectorial a la que pertenece, de la región en la que opera (todas ellas descritas en la Tabla 1) y de una serie de efectos no observador que se asumen tienen una distribución normal con media cero y varianza igual a la unidad.

$$
y_{i}^{*}=\beta_{1} c_{i}+\beta_{2} s_{i}+\beta_{3} r_{i}+u_{i}
$$

Nótese que dado el carácter binario de nuestra variable dependiente utilizamos modelos de regresión probit para nuestro análisis.

Finalmente, con el objetivo de responder a nuestra pregunta de investigación, que planteaba cómo el nivel de cooperación tecnológica regional condiciona la influencia de los factores asociados con la probabilidad de abandono, para cada una de las 24 provincias del Ecuador se calculó el porcentaje de empresas que, durante el periodo 2012-2014, cooperó con otras empresas (clientes, proveedores y competidores) o instituciones de investigación (consultores, universidades, laboratorios y empresas de $\mathrm{I}+\mathrm{D}$, organismos públicos de ciencia y tecnología) en al menos una de las siguientes actividades de innovación: $\mathrm{I}+\mathrm{D}$, ingeniería y diseño, capacitación, asistencia técnica, información, pruebas de productos y financiamiento. Posteriormente, en base a la mediana del nivel de cooperación tecnológica regional, se divide la muestra para empresas que se encuentran en regiones por debajo de la mediana y empresas que se encuentran en regiones por encima de la mediana. El Apéndice 2 muestra el nivel de cooperación tecnológica en cada una de las 24 provincias del Ecuador, agrupadas por regiones de alto y bajo nivel de cooperación tecnológica.

\section{Resultados}

4.1. Factores asociados con el abandono de actividades de innovación La columna (1) de la Tabla 2 muestra los resultados de la regresión probit sobre los factores asociados con la probabilidad de abandono de actividades de innovación para toda la muestra, mientras que la columna (2) presenta los efectos marginales.

\begin{tabular}{|c|c|c|}
\hline & (1) & (2) \\
\hline \multirow[t]{2}{*}{ Mediana } & $-0.319^{* * *}$ & $-0.056^{* * *}$ \\
\hline & $(0.129)$ & \\
\hline \multirow[t]{2}{*}{ Grande } & -0.055 & -0.011 \\
\hline & $(0.077)$ & \\
\hline \multirow[t]{2}{*}{ Int_exportación } & $0.368^{* *}$ & $-0.076^{\star *}$ \\
\hline & $(0.169)$ & \\
\hline \multirow[t]{2}{*}{ Edad } & -0.003 & -0.000 \\
\hline & $(0.002)$ & \\
\hline \multirow[t]{2}{*}{ Solo_id } & $0.346^{*}$ & $0.085^{*}$ \\
\hline & $(0.191)$ & \\
\hline \multirow[t]{2}{*}{ Solo_otros } & $0.147^{* *}$ & $0.031^{\star *}$ \\
\hline & $(0.075)$ & \\
\hline \multirow[t]{2}{*}{ Id_otros } & $0.485^{\star * *}$ & $0.119^{* * *}$ \\
\hline & $(0.092)$ & \\
\hline \multirow[t]{2}{*}{ Coop_empresas } & 0.056 & 0.012 \\
\hline & $(0.098)$ & \\
\hline \multirow[t]{2}{*}{ Coop_instituciones } & 0.170 & 0.038 \\
\hline & $(0.160)$ & \\
\hline \multirow[t]{2}{*}{ Coop_ambas } & $0.262^{* * *}$ & $0.057^{* * *}$ \\
\hline & $(0.102)$ & \\
\hline Efectos fijos sectoriales & Incluidos & Incluidos \\
\hline Efectos fijos regionales & Incluidos & Incluidos \\
\hline Número de observaciones & 2586 & \\
\hline Pseudo R2 & 0,065 & \\
\hline
\end{tabular}

Tabla 2. Factores asociados con el abandono de actividades de innovación

(Notas: (1) Errores estándar robustos entre paréntesis. $(2)^{* * *},{ }^{* *},{ }^{*}=$ significancia estadística al 99\%, 95\% y 90\%. (3) La estimación incluye una constante.)

En primer lugar, los resultados de la Tabla 2 señalan que son las empresas de mediano tamaño las que, en comparación con las pequeñas, tienen una menor probabilidad de abandonar sus actividades de innovación; mientras que las grandes no muestran diferencias significativas. Este resultado sugiere que las ventajas asociadas con el tamaño se dan hasta un cierto número de empleados, a partir del cual la excesiva carga burocrática de las grandes empresas dificulta la toma de decisiones, lo que hace que las empresas de gran tamaño tengan la misma probabilidad de abandonar sus actividades de innovación que las pequeñas. 
En segundo lugar, se observa que aquellas empresas con mayor intensidad exportadora tienen una mayor probabilidad de abandonar sus actividades de innovación. Esto podría sugerir que, debido a que las empresas de los países en desarrollo tienen baja capacidad de absorción, la internalización de conocimientos y tecnologías externas, en lugar de facilitar el desarrollo de actividades de innovación lo complejiza, lo que acaba por aumentar la probabilidad de abandono. Estos resultados concuerdan con los encontrados por García-Vega y López (2010) quienes identificaron que las empresas exportadoras tenían una mayor probabilidad de abandonar actividades de innovación. Sin embargo, Castro et al. (2017) evidenciaron exactamente lo contrario.

En tercer lugar, se puede observar que la edad de la empresa no se asocia significativamente con la probabilidad de abandono. Una posible explicación a este resultado podría ser que la experiencia de la empresa solo se manifiesta en una mayor eficacia en el desarrollo de actividades de innovación cuando el sistema nacional de innovación está lo suficientemente avanzado. En consecuencia, cuando los sistemas de innovación están en una fase emergente, las empresas todavía no han sido capaces de desarrollar las suficientes capacidades tecnológicas como para que su experiencia en el desarrollo de proyectos de innovación se materializa en una mayor tasa de éxito.

Respecto a las variables que reflejan el tipo de actividades de innovación en las que invierten las empresas; en primer lugar, tal y como era de esperar, las empresas que invierten en actividades de innovación, independientemente del tipo de actividades, muestran una mayor probabilidad de abandono que aquellas empresas que sin invertir en actividades de innovación introducen nuevas tecnologías en el mercado. En segundo lugar, los efectos marginales de los coeficientes de estas variables sugieren que las empresas que mayor probabilidad de abandonar sus actividades de innovación son las empresas que simultáneamente invierten en I+D y en otras actividades de innovación, seguidas de las que solo invierten en $\mathrm{I}+\mathrm{D}$ y, por último, de las que solo invierten en otras actividades de innovación. En consecuencia, estos resultados sugieren que cuando la actividad innovadora es de mayor complejidad y requiere de inversiones en $\mathrm{I}+\mathrm{D}$, la probabilidad de que las empresas, de un país en desarrollo, se vean forzadas a abandonar sus proyectos de innovación aumenta. En consecuencia se confirma la hipótesis H.1. De hecho nuestros resultados no solo confirman la hipótesis sino que indican que las empresas que mayor probabilidad de abandono presentan son aquellas que combinan en su proyecto innovador inversiones en I+D con inversiones en otras actividades de innovación; sugiriendo que la probabilidad de abandono es mayor en las empresas que desarrollan proyectos de innovación de mayor complejidad tecnológica.

En relación a las variables de cooperación que distinguen entre las empresas que cooperan solo con otras empresas (Coop_empresas), de las que lo hacen solo con instituciones de investigación (Coop_instituciones) y de las que combinan a ambos tipos de socios (Coop_ambas), los resultados indican que solo las empresas que combinan ambos tipos de socios tienen una mayor probabilidad de abandonar sus proyectos de innovación que aquellas empresas que no cooperan. De hecho, este resultado debería ser tomado con cautela puesto que el coeficiente es solo significativo al 90\%. En consecuencia, estos resultados no permiten dar respaldo a la hipótesis H.2, que consideraba que, en un contexto de país en desarrollo, las empresas que cooperan con instituciones de investigación tienen una mayor probabilidad de abandono que las empresas que no cooperan o que cooperan solo con otras empresas. De hecho, nuestros resultados indican que las empresas que cooperan exclusivamente con instituciones de investigación no tienen una mayor probabilidad de abandonar sus actividades de innovación que las empresas que no cooperan. Una posible explicación al resultado de que las empresas que cooperan con ambos tipos de socios tengan mayor probabilidad de abandonar sus actividades de innovación podría ser que cuando las empresas, de un país en desarrollo, tratan de integrar en un mismo proyecto innovador a agentes con lógicas distintas, esto acaba por complejizar el proyecto de innovación, lo que incrementa la probabilidad de abandono.

\subsection{Cooperación tecnológica regional y abandono de actividades de innovación}

La Tabla 3, muestra los resultados de los modelos probit (columnas 1 y 3 ) y los efectos marginales (columnas 2 y 4 ) para las sub-muestras de empresas localizadas en provincias con un nivel de cooperación tecnológica inferior a la mediana (Cooperación baja) y para las localizadas en provincias con un nivel de cooperación tecnológica superior a la mediana (Cooperación alta). Además se presentan los efectos marginales de ambas estimaciones.

Tabla 3. Factores asociados con la probabilidad de abandono en función del nivel de cooperación tecnológica regional

\begin{tabular}{|c|c|c|c|c|}
\hline & \multicolumn{2}{|c|}{ Cooperación baja } & \multicolumn{2}{|c|}{ Cooperación alta } \\
\hline & (1) & (2) & (3) & (4) \\
\hline Mediana & $\begin{array}{l}-0.206 \\
(0.241)\end{array}$ & -0.026 & $\begin{array}{c}-0.400^{* * *} \\
(0.157)\end{array}$ & $-0.081^{* * *}$ \\
\hline Grande & $\begin{array}{c}0.191 \\
(0.146)\end{array}$ & 0.028 & $\begin{array}{l}-0.170^{*} \\
(0.094)\end{array}$ & $-0.041^{*}$ \\
\hline Int_exportación & $\begin{array}{c}0.564^{* * *} \\
(0.240)\end{array}$ & $0.080^{* * *}$ & $\begin{array}{c}0.143 \\
(0.256)\end{array}$ & 0.035 \\
\hline Edad & $\begin{array}{l}-0.001 \\
(0.004)\end{array}$ & -0.000 & $\begin{array}{l}-0.004 \\
(0.003)\end{array}$ & -0.001 \\
\hline Solo_id & $\begin{array}{l}0.833^{* *} \\
(0.267)\end{array}$ & $0.193^{* *}$ & $\begin{array}{l}-0.117 \\
(0.273)\end{array}$ & -0.027 \\
\hline Solo_otros & $\begin{array}{l}0.230 \\
(0.150)\end{array}$ & 0.035 & $\begin{array}{c}0.106 \\
(0.088)\end{array}$ & 0.026 \\
\hline Id_otros & $\begin{array}{c}0.611^{\star * *} \\
(0.154)\end{array}$ & $0.114^{* * *}$ & $\begin{array}{c}0.422^{* * *} \\
(0.118)\end{array}$ & $0.117^{\star * *}$ \\
\hline Coop_empresas & $\begin{array}{l}0.127 \\
(0.172)\end{array}$ & 0.018 & $\begin{array}{l}-0.007 \\
(0.126)\end{array}$ & -0.002 \\
\hline Coop_instituciones & $\begin{array}{c}0.304 \\
(0.251)\end{array}$ & 0.052 & $\begin{array}{c}0.083 \\
(0.212)\end{array}$ & 0.021 \\
\hline Coop_ambas & $\begin{array}{c}0.497^{* * *} \\
(0.180)\end{array}$ & $0.083^{* * *}$ & $\begin{array}{c}0.147 \\
(0.132)\end{array}$ & 0.037 \\
\hline $\begin{array}{l}\text { Efectos fijos } \\
\text { sectoriales }\end{array}$ & Incluidos & Incluidos & Incluido & Incluidos \\
\hline $\begin{array}{l}\text { Efectos fijos } \\
\text { regionales }\end{array}$ & Incluidos & Incluidos & Incluido & Incluidos \\
\hline $\begin{array}{l}\text { Número de } \\
\text { observaciones }\end{array}$ & 969 & & 1600 & \\
\hline Pseudo R2 & 0.047 & & 0.1021 & \\
\hline
\end{tabular}

(Notas: (1) Errores estándar robustos entre paréntesis. $(2)^{* * *},{ }^{* *},{ }^{*}=$ significancia estadística al 99\%, 95\% y 90\%. (3) Todas las estimaciones incluyen una constante.) 
Tal y como se puede observar, en términos generales, los resultados de la Tabla 3 indican que los factores que se asocian con el abandono de actividades de innovación difieren ampliamente en función del nivel de cooperación tecnológica regional. En concreto, los resultados indican que en las regiones donde existe un bajo nivel de cooperación, el tamaño no se asocia con la probabilidad de abandono; mientras que en las regiones con alto nivel de cooperación, tanto las medianas como las grandes empresas tienen una menor probabilidad de abandono que las pequeñas (aunque para las grandes el coeficiente es solo significativo al 90\%). En consecuencia, estos resultados sugieren que, para que el tamaño suponga una ventaja en la puesta en marcha de actividades de innovación, es necesario que las empresas estén localizadas en determinados contextos regionales, caracterizados por una mayor capacidad para organizar procesos de aprendizaje colectivos a través de relaciones de cooperación tecnológica. Adicionalmente, nótese que para el caso de las regiones con alto nivel de cooperación, las empresas medianas siguen teniendo una menor probabilidad de abandono que las grandes.

Respecto a la intensidad de la exportación, los resultados indican que en las regiones de bajo nivel de cooperación tecnológica, la intensidad de la exportación se asocia positivamente con probabilidad de abandono. Sin embargo, en el caso de las localizadas en regiones con alto nivel de cooperación, esta variable deja de ser significativa. Esto sugiere que para empresas exportadoras sean capaces de integrar satisfactoriamente los conocimientos y tecnologías provenientes de los mercados internacionales requieren de estar en regiones caracterizadas por mayores niveles de cooperación; de lo contrario tendrán una mayor probabilidad de abandonar sus actividades de innovación.

En relación al tipo de actividades de innovación en las que se invierte, los resultados indican que, independientemente del nivel de cooperación tecnológica regional, las empresas que invierten simultáneamente en $\mathrm{I}+\mathrm{D}$ y en otras actividades de innovación muestran una mayor probabilidad de abandono; presumiblemente debido al mayor nivel de complejidad de estos proyectos de innovación. Sin embargo, las empresas que solo invierten en $\mathrm{I}+\mathrm{D}$ tienen solo una mayor probabilidad de abandono si se localizan en regiones con un bajo nivel de cooperación tecnológica. En consecuencia, estos resultados sugieren que en las regiones en las que existe un mayor intercambio de información a través de relaciones de cooperación, las empresas tienen una mayor probabilidad de ser exitosas en sus proyectos de innovación que se basan en inversiones en actividades de I+D. Sin embargo, las empresas que invierten $\mathrm{I}+\mathrm{D}$ en regiones caracterizadas por un bajo nivel de cooperación tecnológica tienen una mayor probabilidad de abandonar dichas inversiones. Esto sugiere que, en los países en desarrollo, la puesta en marcha de actividades de $\mathrm{I}+\mathrm{D}$ requiere de un determinado contexto institucional que permita el acceso a información y recursos complementarios, de manera tal que las empresas puedan finalizar con éxito sus proyectos de innovación. Finalmente, en relación a las empresas que solo invierten en otros actividades para la innovación, los resultados indican que éstas no tienen una mayor probabilidad de abandonar sus actividades de innovación, independientemente del contexto regional en las que operen.
Respecto a las variables de cooperación, los resultados indican que en contextos regionales caracterizados por un bajo nivel de cooperación, solamente las empresas que cooperan simultáneamente con empresas e instituciones tienen mayor probabilidad de abandonar proyectos de innovación. Sin embargo, las empresas que cooperan con ambos tipos de socios, localizadas en regiones caracterizadas por un alto nivel de cooperación no son más propensas a abandonar sus actividades de innovación. Por tanto, estos resultados sugieren que en las regiones en las que las empresas son más propensas a organizar procesos de aprendizaje colectivo, las relaciones de cooperación tecnológica son mucho más efectivas.

\section{Conclusiones}

Utilizando datos de la Encuesta Nacional de Actividades de Innovación del Ecuador (ENAI, 2015), este trabajo analiza los factores que se asocian con la probabilidad de abandono de actividades de innovación en un país en desarrollo. Adicionalmente, se examina cómo el nivel de cooperación tecnológica, que existe en las regiones, en las que se localizan las empresas, condiciona la influencia de dichos factores.

Los resultados sugieren que, en Ecuador, son las empresas de mediano tamaño las que tienen una menor probabilidad de abandonar sus actividades de innovación. Además, se observa que la intensidad de la exportación está positivamente asociada con la probabilidad de abandono; lo que sugiere que cuando las empresas exportadoras no tienen la suficiente capacidad de absorción, como para internalizar satisfactoriamente los conocimientos provenientes de otros mercados, esto puede dificultar la puesta en marcha de actividades de innovación. Los resultados también indican que cuanto mayor es la complejidad tecnológica de los proyectos de innovación en los que invierten las empresas, mayor es la probabilidad de abandono. Esto es así puesto que, en comparación con las empresas innovadoras que no invierten en actividades de innovación, las que muestran una mayor probabilidad de abandono son las que combinan inversiones tanto en $\mathrm{I}+\mathrm{D}$ como en otras actividades de innovación, seguidas de las que solo invierten en $\mathrm{I}+\mathrm{D}$ y seguidas de las que solo invierten en otras actividades de innovación. Adicionalmente, observamos que las empresas que cooperan simultáneamente con otras empresas e instituciones de investigación son las que muestran una mayor probabilidad de abandono; lo sugiere que las empresas de los países en desarrollo tienen dificultades para integrar en el mismo proyecto a socios con lógicas distintas y que las que lo hacen muestran mayores tasas de abandono.

Respecto a la influencia del nivel de cooperación tecnológica regional, los resultados muestran, en primer lugar, que el tamaño de la empresa solo ofrece una ventaja de cara al desarrollo de actividades de innovación en las regiones caracterizadas por un alto nivel de cooperación tecnológica. En segundo lugar, se observa que las empresas exportadoras solo muestran una mayor probabilidad de abandono en aquellas regiones caracterizadas por un bajo nivel de cooperación. En tercer lugar, observamos que en las regiones de mayor nivel de cooperación tecnológica, las empresas son capaces de desarrollar con éxito proyectos de innovación de mayor complejidad. Aunque, inde- 
pendientemente del nivel de cooperación regional, aquellas empresas que invierten tanto en actividades de $\mathrm{I}+\mathrm{D}$ como en otras actividades de innovación son más propensas a abandonar sus proyectos de innovación. Finalmente, los resultados indican que en las regiones de bajo nivel de cooperación las empresas tienen dificultades para integrar en un mismo proyecto innovador a otras empresas con instituciones de investigación; mientras que en las regiones de alto nivel de cooperación las empresas que cooperan no muestran una mayor ni menor probabilidad de abandono que las que no cooperan.

Estos resultados tienen importantes implicaciones de cara a la implementación de políticas tecnológicas en los países en desarrollo; dado que sugieren que aquellas políticas a nivel regional que traten de fomentar la cooperación tecnológica entre empresas e instituciones de investigación pueden tener una gran influencia en el éxito innovador de las empresas y, en consecuencia, en posibilitar procesos de catch-up con los países más avanzados. De manera adicional, este artículo abre nuevas líneas de investigación, dado que sería pertinente analizar qué otras características de los sistemas regionales de innovación, más allá del nivel de cooperación tecnológica, condicionan la influencia de los factores asociados con el abandono de actividades de innovación. Por último, es importante enfatizar que los resultados de esta investigación no deben ser interpretados como relaciones causales entre las distintas variables independientes y la probabilidad de abandono de actividades de innovación, sino como meras asociaciones entre las variables. En esta línea, varias de las variables independientes podrían ser endógenas, por lo que sería acertado aplicar otro tipo de metodologías que permitan controlar por el sesgo de endogeneidad.

\section{Referencias}

Arocena, R., \& Sutz https: //onlinelibrary.wiley.com/doi/pdf/10.1002/ mde.4090100203, J. (2001). Innovation systems and developing countries. DRUID Working Paper 02-05. Copenhagen: Danish Research Unit for Industrial Dynamics. Doi: 10.2307/1515847

Asheim, B., \& Gertler. (2005). The geography of innovation: regional innovation systems. En I. Fagerberg, D. Mowery, \& R. Nelson, The oxford handbook of innovation (págs. 291-317). New York: Oxford University Press.

Barge-Gil, A., Nieto, M., \& Santamaría, L. (2011). Hidden innovators: The role of NON-R\&D activities. technology analysis \& strategic management 23:4, 415-432.

Becker, B., \& Hall, S. (09 de 2013). Do R\&D strategies in high-tech sectors differ from those in low-tech sectors? An alternative approach to testing the pooling assumption. Economic Change and Restructuring 46, 183-202.

Bender, G., \& Laestadius, S. (2004). Non-science based innovativeness: on capabilities relevant to generate profitable novelty. Policy and Innovation in Low-Tech.

Braczyk, H.-J., Cooke, P., \& Heidenreich, M. (1998). Regional innovation systems. Routledge.
Caniels, M., \& Romijn, H. (2003). Firm-level knowledge accumulation and regional dynamics. Industrial and Corporate Change 12(6), 1253-1278.

Chaminade, C., Lundvall, B., Vang-Laurisen, J., \& Joseph, K. (2009). Innovation policies for development: towards a systematic experimentation-based approach. En Handbook of innovation systems and developing countries: building domestic capabilities in a global setting.

Cincera, M., \& Simeth, M. (2015). Corporate science, inovation and firm Value. Management Science, 1-26. Obtenido de https://ssrn. com/abstract $=2317166$ or http://dx.doi.org/10.2139/ssrn.2317166

Cohen, W. (1995). Empirical studies of innovation activity. En Handbook of the economics of innovation and technological change (págs. 182-237).

Cohen, W., \& Levinthal, D. (1990). Absorptive capacity: A new perspective on learning and innovation. Administrative Science Quarterly $35,128-152$.

Cruz-Castro, L., Holl, A., Rama, R., \& Sanz-Menéndez Luis. (2017). Economic crisis and company R\&D in Spain: do regional and policy factors matter? Industry and Innovation, 1-18. doi: $10.1080 / 13662716.2017 .1355231$

Cuijpers, M., H. Guenter, \& K. Hussinger. 2011. "Costs and benefits of inter-departmental innovation collaboration.” Research Policy 40(4), 565-575.

Fagerberg, J., Mowery, D., \& Nelson, R. (2005). The oxford handbook of innovation. New York: Oxford University Press.

Fernández-Sastre, J., \& Martín Mayoral, F. (2017). Assessing the impact of public support for innovation in an emerging innovation system. International Journal of Technological Learning, Innovation and Development 9(1), 42-64.

Freeman, C., \& Soete, L. (1997). The economics of industrial innovation. Cambridge: MIT Press.

Galia, F., \& Legros, D. (2004). Complementarities between obstacles to innovation: evidence from France. Research Policy 33, 1185-1199.

García-Vega, M., \& López, A. (2010). Determinantes del abandono de actividades de innovación: Evidencia para la empresa española. Cuaderno de Economía y Dirección de la Empresa, 45, 69-92.

Gilly, J.-P., \& Kechidi, M. (2014). Resilience of organizations and territories: The role of pivot firms. European Management Journal 32(4), 596-602.

Grilinches, Z. (1995). R\&D and productivity: econometric results and measurement issues. En P. Stoneman, Handbook of the economics of innovation and technological change (págs. 52-89). Oxford and Cambridge: Wiley-Blackwell. 
Hage, J., G. Jordan, J. Mote, \& Y. Whitestone. 2008. Designing and facilitating collaboration in R\&D: A case study. Journal of Engineering and Technology Management 25(4), 256-268.

Harris, R., \& Moffat, J. (2011). R\&D, innovation and exporting. SERC Discussion Paper 0073. Spatial Economic Research Centre, LSE.

Hemmert, M. (2004). The influence of institutional factors on the technology acquisition performance of high-tech firms: survey results from Germany and Japan. Research Policy, 1019-1039.

Iacovou, C., \& Dexter, A. (2005). Surviving it project Cancellations. Communications of the ACM, Vol. 48, No. 4, 83-86.

Kale, P., Dyer, J., \& Singh, H. (2002). Alliance capability, stock market response, and long-term alliance success: the role of the alliance function. Strategic Management Journal 23, 747-767.

Karlsson, C., \& Tavassoli, S. (2016). Innovation strategies of firms: what strategies and why? The journal of Technological Transfer, Springer, Vol. 41(6), 1483-1506.

Klevorick, A., Levin, R., Nelson, R., \& Winter, S. (1995). On the sources and significances on interindustry differences in technological opportunity. Research Policy 24, 185-205.

López-Bazo, E., \& Montellón, E. (2018). Innovation, heterogeneous firms and the region: evidence from Spain. Regional Studies 52(5), 673-687.

Lundvall, B. (1992). Introduction. En B. Lundvall, National systems of innovation - towards a theory of innovation and interactive learning (págs. 1-19). London: Pinter Publisher.

Lunn, J., \& Martin, S. (1986). Market structure, firm structure and research and development. Quarterly Review of Economics and $\mathrm{Bu}-$ siness 26, 31-44.

Méndez-Morales, E. (2012). Barreras financieras de las empresas innovadoras españolas. Working Paper 2013/09. Madrid: Universidad Autónoma de Madrid, Facultad de Economía.
Mohnen, P., Palm, F., Schim Van Der Loeff, S., \& Tiwari, A. (2008). Financial constraints and other obstacles: are they a threat to innovation activity? De Economist 156, No. 2, 201-214. Doi: 10.1007/ s10645-008-9089-y

Mowery, D., Okley, J., \& Silverman, B. (1996). Strategic alliances and interfirm knowledge transfer. Winter Special Issue, 77-91.

Paunov, C. (2012). The global crisis and firms' investments in innovation. Research Policy 41, 24-35.

Reuer, J., \& Zollo, M. (2005). Termination outcomes of research alliances. Research Policy 34, 101-115.

Santamaría, L., Nieto, M., \& Barge-Gil, A. (2009). Beyond formal $\mathrm{R} \& \mathrm{D}$ : taking advantage of other sources of innovation in low -and medium-technology industries. Research Policy 38 (3), 507 - 517.

Sawang, S., \& Matthews, J. (2010). Positive relationships among collaboration for innovation, past innovation abandonment and future product introduction in manufacturing SMEs. Interdisciplinary Journal of Contemporary Research in Business, 2(6), 106-117.

Saxenian, A. (1994). Regional advantage: culture and competition in silicon valley and route 128. Boston: Harvard University Press.

Shan, W., Walker, G., \& Kogut, B. (1994). Interfirm cooperation and startup innovation in the biotechnology industry, Vol. 15. Strategic Management Journal, 387-394.

Song, X. M., M. M. Montoya-Weiss, \& J. B. Schmidt. 1997. Antecedents and consequences of cross-functional cooperation: A comparison of $\mathrm{R} \& \mathrm{D}$, manufacturing, and marketing perspectives.

$\mathrm{Wu}$, Y. (2015). Organizational structure and product choice in knowledge-intensive firms. management science. doi:https://doi. org/10.1287/mnsc.2014.2080

Zouaghi, F., \& Sánchez, M. (2016). Has the global financial crisis had different effects on innovation performance in the agri-food sector by comparison to the rest of the economy? Trends in Food Science \& Technology 50, 230-242. 


\section{Apéndice}

Apéndice 1. Estadísticos descriptivos y correlaciones de las variables a nivel empresa

\begin{tabular}{|c|c|c|c|c|c|c|c|c|c|c|c|c|c|c|}
\hline 1 & Pequeña & 0.454 & 0.498 & 1 & & & & & & & & & & \\
\hline 3 & Grande & 0.453 & 0.498 & -0.831 & -0.290 & 1 & & & & & & & & \\
\hline 4 & Int_exportación & 0.050 & 0.186 & -0.156 & 0.022 & 0.143 & 1 & & & & & & & \\
\hline 6 & Solo_id & 0.028 & 0.165 & -0.022 & 0.011 & 0.016 & 0.017 & 0.076 & 1 & & & & & \\
\hline 7 & Solo_otros & 0.382 & 0.486 & -0.030 & 0.033 & 0.011 & -0.051 & -0.035 & -0.133 & 1 & & & & \\
\hline 8 & Id_otros & 0.161 & 0.368 & -0.098 & -0.023 & 0.112 & 0.069 & 0.095 & -0.074 & -0.345 & 1 & & & \\
\hline 9 & Coop_empresas & 0.470 & 0.499 & 0.139 & -0.040 & -0.115 & -0.039 & -0.075 & -0.004 & -0.011 & -0.082 & 1 & & \\
\hline
\end{tabular}

(Notas: (1) correlaciones en negrita significativas al 95\%.)

Apéndice 2. Nivel de cooperación tecnológica regional

\begin{tabular}{lc}
\hline Provincia & Nivel de cooperación \\
\hline Cooperación baja & \\
Morona Santiago & 0,040 \\
Zamora Chinchipe & 0,071 \\
Galápagos & 0,080 \\
El Oro & 0,143 \\
Santa Elena & 0,162 \\
Loja & 0,205 \\
Azuay & 0,222 \\
Cañar & 0,222 \\
Los ríos & 0,236 \\
Manabí & 0,271 \\
Guayas & 0,275 \\
Santo domingo & 0,302 \\
\hline Cooperación alta & \\
Carchi & 0,350 \\
Napo & 0,423 \\
Bolívar & 0,440 \\
Pastaza & 0,464 \\
Esmeraldas & 0,480 \\
Orellana & 0,492 \\
Chimborazo & 0,503 \\
Sichincha & 0,512 \\
Imbabura & 0,516 \\
Cotopaxi & 0,527 \\
\hline & 0,585 \\
\hline$n g u r a h u a$ & 0,589 \\
\hline
\end{tabular}


